\title{
Longer pre-hospital delay in acute myocardial infarction in women because of longer doctor decision time
}

Jelte Bouma, Jan Broer, Jan Bleeker, Eric van Sonderen, Betty Meyboom-de Jong, Mike J L DeJongste

\section{Study} objective-To measure the prehospital delay times in patients with proven acute myocardial infarction (AMI) and to identify possibilities for reduction of treatment delay. Design-Descriptive three centre study. Setting-One university teaching hospital and two regional hospitals in Groningen, the Netherlands.

Patients-400 consecutive confirmed AMI patients, age below 75 years, admitted to coronary care departments.

Main results-Mean age was 59 years and $78 \%$ of patients were men. Within two hours after onset of symptoms half of the patients with AMI arrived at the hospital. Patient, doctor, and ambulance delay times (median values) were 30,38 , and 35 minutes respectively. Calling the personal general practitioner (GP) or the locum tenens and whether or not the AMI occurred during a weekend or on a delay times. At night patients waited longer before calling a GP than in the daytime. There was a positive correlation between patient and doctor delay. Twenty two per cent of AMI patients waited two hours or more before calling a GP. Total pre-hospital delay times differed between men and women. Longer doctor delay in women (36 minutes for men and 52 minutes for women) was caused by displacement of specific symptoms, in particular in women. AMI patients who were alone during onset of symptoms showed higher patients delay ( 72 compared with 23 minutes).

Conclusion-In hospital admitted patients younger than 75 years pre-hospital delay times are within acceptable limits. In some subgroups further reduction is attainable, for example in patient delay outside office hours and when patients are alone during onset of symptoms, in doctor delay in cases where women present with symptoms suggestive for AMI. Improvement of facilities for prehospital electrocardiographic diagnosis may facilitate decision making by GPs. Good opportunities for further reduction of treatment delay exist in shortening of hospital delay.

(F Epidemiol Community Health 1999;53:459-464)
The chance to survive an acute myocardial infarction (AMI) is related to fast restoration of coronary blood flow and treatment of complications. ${ }^{1}$ The improvement in survival rates of hospitalised AMI patients is no doubt largely the result of this development. Both improved treatment and a decreasing prevalence of cardiovascular risk factors resulted in reduction of coronary heart disease mortality rates by a third since the late 1960 s. $^{2}{ }^{3}$ Besides reduced mortality, the early start of thrombolytic treatment considerably reduces physical damage of the heart. ${ }^{4}$ The best results are obtained when the treatment is started within the first two hours after AMI..$^{5-7}$ This means that when patients or doctors do not succeed in early recognition of AMI, avoidable damage may occur ("time is tissue"). Total treatment delay includes patient, doctor, ambulance, ${ }^{5-10}$ and hospital delay. ${ }^{11}$ Some early reports indicated patient delay to be the most important component of total delay in hospital admission. ${ }^{12-14}$ A study by Hart, ${ }^{15}$ which was carried out in Rotterdam in the Netherlands in 1978, confirmed this finding. Since that time, the media have published much information about the importance of recognition and early treatment of AMI. In 1990 and 1991 a survey was undertaken in the city of Rotterdam. ${ }^{16}$ From that study it appeared that the patients with AMI called for help much faster as compared with the 1978 study. In 1992 the contribution of the general practitioner (GP) delay to the total prehospital delay seemed to be larger than the patients' share. For a better understanding of the situation it should be stated here that in the Dutch health care system the GP has the role of "gate-keeper". ${ }^{16}$ Therefore, in the Netherlands, most AMI patients are referred to hospital by a GP. The ambulance dispatch centre will meet demands for help after a direct call of the patient or on behalf of the patient if the situation is perceived as life threatening. In the densely populated Netherlands almost all villages with a size of 2000 or more people have a GP living in that same village. Ambulances are stationed in decentralised garages dispersed over cities and small towns according to need. To find out whether or not action should be undertaken to reduce treatment delay in AMI patients, a research project was started in the city of Groningen and its surrounding (population catchment area 400000 people). The aim of this study was to establish the (determinants of) patient, doctor, and ambulance delay times in 
AMI patients in the Groningen area. In this article the different delay times are described in relation to clinical, psychological, geographical, sociodemographic, and time related variables.

\section{Methods}

Between January 1993 and July 1995400 consecutive AMI patients were included. The patients were admitted to the university hospital or a regional hospital in the city of Groningen (170 000 inhabitants). Information about all patients under suspicion of AMI who were transported to hospital was obtained from the ambulance dispatch centre. One day after admission, the hospital was contacted to verify the diagnosis AMI and to gather information regarding admission of other patients, who had arrived using other means of transportation, such as private cars. AMI was diagnosed on basis of a typical enzyme pattern with a peak creatine phosphokinase (CPK) release exceeding twice the normal limit and electrocardiographic signs indicative of evolving AMI. Patients were included when they were younger than 75 years, had developed the AMI outside hospital, the AMI occurred less than 72 hours before, and were able to communicate and to remember what happened. When patients agreed to participate in the study, a written informed consent was signed. Some $6.2 \%$ of the patients who were approached decided not to participate in the study. The interviews took place in the hospital in a separate room in the first week after admission. The structured interviews were conducted by two experienced interviewers, and lasted about 45 minutes. Presence of AMI symptoms (range $0-6$ ), intensity of pain (range $0-4$ ), and the tendency to find support after the initial complaints began were registered on specific checklists. The psychological defence mechanism displacement (yes/no) was based on a checklist that attributes initial cardiac complaints to other organ systems. Sum scores were computed by adding up single item answers. With the Patient Delay Questionnaire ${ }^{17}$ information was collected concerning the time of the onset of the AMI, the time when the GP was called, the time that elapsed before the GP arrived at the patient, and the time at which the ambulance was called by the GP. The delay times as reported by the patients were compared with the information derived from the spouse, partner or a close friend who was well informed about the patient and their situation. The ambulance delay times were collected from the ambulance dispatch centre, which keeps a protocolled case registration for each transported patient. This registration is required by law.

In this study patient delay is defined as the interval between the onset of symptoms and the call for medical help by the patient or by someone on behalf of the patient. For the analysis the patient delay times were divided in quartiles. The lowest quartile ( $<10$ minutes) was called "short delay" and the highest quartile ( $>2$ hours) was called "long delay". The within group was called "medium delay. The doctor delay includes the time between the call for help and the call for an ambulance. Ambulance delay is the interval between the call for the ambulance and the patients' arrival at the hospital.

Doctor delay is subdivided in "travel time to the patient", which can be defined as the time that elapses between the first call for help and the first personal contact between doctor and patient, and diagnosis time of the GP (the time between the first contact and calling the ambulance by the GP). Ambulance delay is subdivided in "travel time to the patient" and "transport time of the patient to the hospital". The latter includes all actions aimed at stabilising or recovering the patient (on the spot or in the ambulance) and the time it takes to transport the patient from the spot of the incident to the hospital. Patients who were referred to a hospital by a GP are called "GP group". Those who directly called the ambulance are mentioned "direct call group".

Information regarding the size of the infarct and patient's medical history was recorded from hospital records. Data included CPK maximum values, sum of the ST segment deviations on the admission electrocardiogram ${ }^{18}$ as indicators of size of AMI and Killip class.

ANALYSIS

Because of the skewed distributions of the delay times, with sometimes large outliers, median values are used. For the same reason non-parametric tests were applied (KruskalWallis one way analysis of variance, MannWhitney-Wilcoxon rank sum W test, median

Table 1 Characteristics of patient group ( $n=400$; valid per cent in parentheses)

\begin{tabular}{lrl}
\hline Age (y) & & \\
$35-44$ & 41 & $(10)$ \\
$45-54$ & 89 & $(22)$ \\
$55-64$ & 141 & $(35)$ \\
65-74 & 129 & $(32)$ \\
Sex (male) & 311 & $(78)$ \\
Education & & \\
elementary or lower schooling & 281 & $(71)$ \\
secondary schooling & 60 & $(15)$ \\
higher professional training, college or & & \\
university & 53 & $(13)$ \\
Domicile (urban area) & 160 & $(40)$ \\
Route to hospital via & & \\
own GP & 165 & $(41)$ \\
locum tenens & 189 & $(47)$ \\
emergency call & 40 & $(11)$ \\
other & 3 & $(1)$ \\
Hospital & & \\
university hospital & 204 & $(51)$ \\
regional hospitals & 196 & $(49)$ \\
Within office hours (yes) & 168 & $(42)$ \\
First AMI (yes) & 350 & $(88)$ \\
Number of specific complaints & & \\
0 & 34 & $(9)$ \\
1 & 122 & $(31)$ \\
2 & 117 & $(29)$ \\
3 & 73 & $(18)$ \\
$4-7$ & 54 & $(14)$ \\
Intensity of pain & & \\
0 & 111 & $(31)$ \\
1 & 95 & $(27)$ \\
2 & 66 & $(19)$ \\
4 & 70 & $(20)$ \\
missing & 14 & $(4)$ \\
Killip class & 44 & - \\
1 & & \\
2 & 312 & $(83)$ \\
3 & 49 & $(13)$ \\
4 & 6 & $(1,6)$ \\
missing & 9 & $(2,4)$ \\
\hline & & \\
\hline
\end{tabular}


Table 2 Delay times in minutes in the Groningen area, median values $(n=400)$

\begin{tabular}{lcl}
\hline Type of delay & Median & $\begin{array}{l}25 \text { and } 75 \\
\text { Percentile }\end{array}$ \\
\hline Patient delay & 30 & $10-90$ \\
Doctor delay (total) & $38^{\star}$ & $22-77$ \\
$\quad$ doctor delay: to patient & 11 & $6-16$ \\
$\quad$ doctor delay: diagnosis time & 24 & $10-55$ \\
Ambulance delay (total) & 35 & $30-45$ \\
$\quad$ ambulance delay: to patient & 9 & $6-13$ \\
ambulance delay: patient to hospital & 27 & $23-33$ \\
Total pre-hosptial delay & 111 & $72-250$ \\
\hline
\end{tabular}

^Sub-totals do not add up to arithmetic totals because of the use of median times.

test). Alpha was set at 0.05, two sided. After logarithmic transformation of skewed delay times were normally distributed. Analysis of variance was performed on logarithmic transformed time variables controlling for the effect of age and sex.

\section{Results}

Table 1 shows the characteristics of the participating patients. The average age was about 59 years, $78 \%$ was men. For the vast majority $(88 \%)$ this was the first AMI; $20 \%$ of patients had a history of previous cardiac disease (angina pectoris, AMI, angiography, bypass graft). Ninety one per cent of the patients had one or more specific complaints. Patients were referred to the hospital by their GP in $41 \%$, by a locum tenens $47 \%$ and $11 \%$ of AMI patients directly called the emergency number for an ambulance.

In table 2 the different segments of median delay times are depicted. The total delay time was 111 minutes. Patient, doctor, and ambulance delay times were 30, 38, and 35 minutes respectively. When called for help, $50 \%$ of the GPs arrived at the patient within 11 minutes; the median time to decide whether admission to a hospital was necessary was 24 minutes.
KEY POINTS

- Within two hours after onset of symptoms half of the patients with AMI arrived at the hospital.

- Patient, doctor, and ambulance were equally quick, with delay times of 30,38 , and 35 minutes respectively (median values).

- Living in an urban or rural area had no consequences for delay times.

- Outside office hours patients waited longer before calling a GP then within office hours.

- Total delay times between men and women differed significantly. This was mainly caused by doctor delay (36 minutes for men and 52 minutes for women).

Half of the ambulances arrived within nine minutes at the patient; the stabilisation and transport time of the patients to the hospital was 27 minutes.

The GP group was divided, according to the highest and lowest quartile of the patient delay times, in a short $(n=109,<10$ minutes, median value 5 minutes), long ( $n=89,>2$ hours, median value 6 hour and 15 minutes) and a medium patient delay group $(n=156$, between 10 minutes and 2 hours, median value $30 \mathrm{~min}$ utes). In figure 1 the corresponding doctor and ambulance delay times are shown.

The travel time of the GP, the time the ambulance needed to arrive at the patient as well the transport time to the hospital were almost identical for the three groups. Significant differences existed however in the diagnosis time of the doctor for the three patient delay subgroups. The longer the patient had waited the more time the GP needed to decide to call

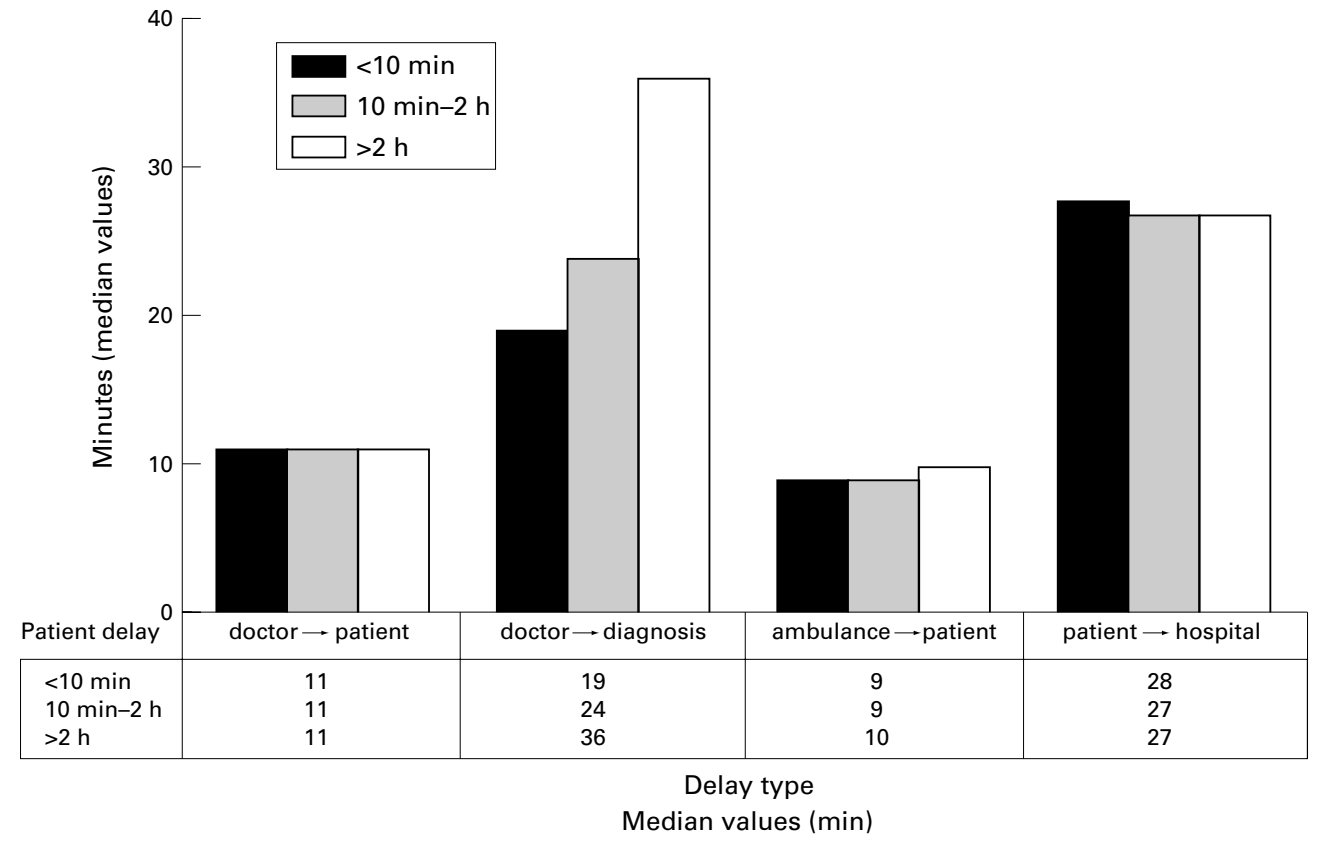

Figure 1 Doctor and ambulance delay of patients with a short, medium, and long patient delay. 


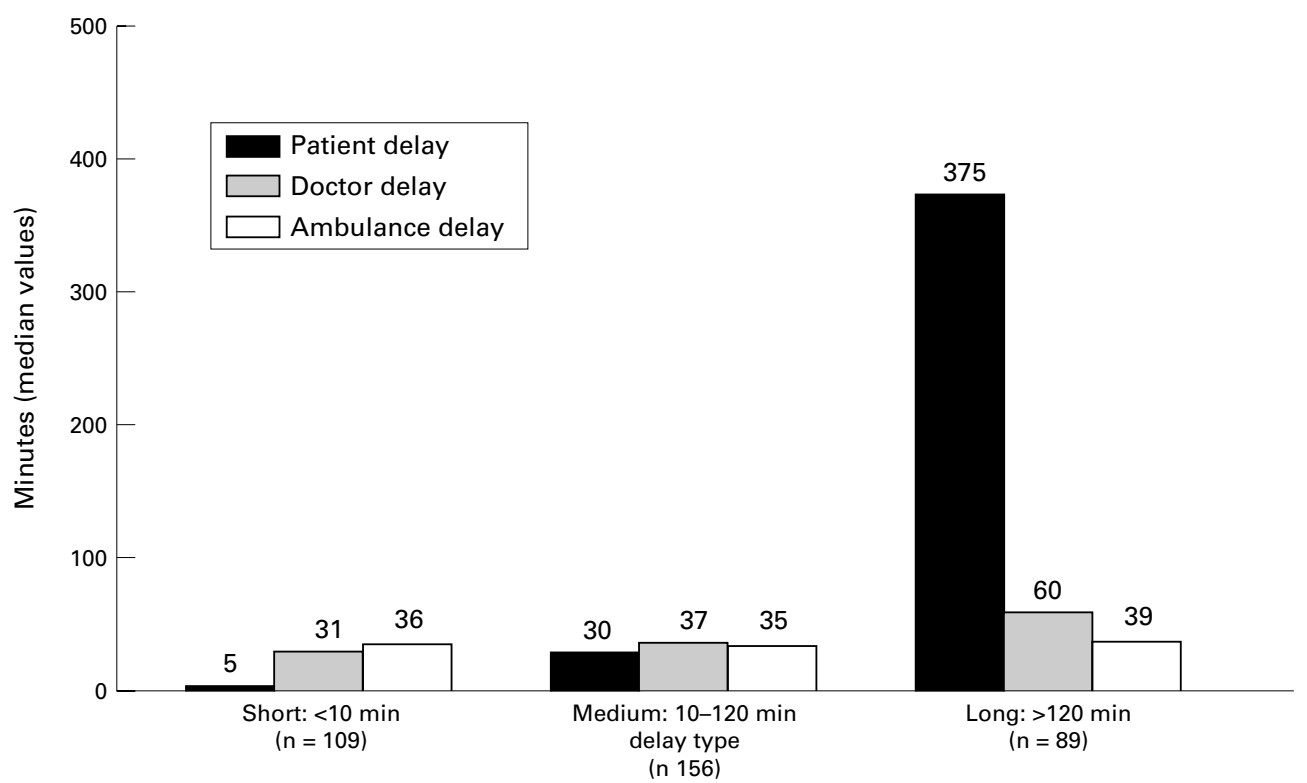

Figure 2 Patient, doctor, and ambulance delay according to patient delay.

an ambulance (Kruskal-Wallis one way analysis of variance, $p=0.0009$ ).

In figure 2 all delay times are depicted, showing clearly the relative importance of the three different (patient) delay groups. In the long patient delay sub-group patient delay is decisive for total pre-hospital delay.

In table 3 an overview is given of the relation between median patient, doctor, ambulance, and total pre-hospital delay and some relevant variables for the patients who were admitted to hospital after consultation of a GP. Total delay of men was 115 minutes and women scored 135 minutes (median values). Patients with signs of left ventricular failure (Killip class $>1$ ) had a significant shorter patient delay. Doctor delay was substantial longer in women (52 minutes) compared with men (36 minutes). This was caused by a longer diagnosis time of the GP in women. The sex difference in doctor delay could not be explained by age, intensity of pain, specificity of complaints, and Killip class (one way analysis of variance: $F=4.38$;

Table 3 Median patient doctor, ambulance delay and total delay times for some relevant variables for the general practitioner group $(n=354)$

\begin{tabular}{|c|c|c|c|c|}
\hline & $\begin{array}{l}\text { Patient } \\
\text { delay }\end{array}$ & $\begin{array}{l}\text { Doctor } \\
\text { delay }\end{array}$ & $\begin{array}{l}\text { Ambulance } \\
\text { delay }\end{array}$ & Total delay \\
\hline \multicolumn{5}{|l|}{ Sex } \\
\hline Male & 28 & 36 & 35 & $1 \mathrm{~h} 55 \mathrm{~min}$ \\
\hline Female & 30 & $52^{\star \star}$ & 38 & $2 \mathrm{~h} 15 \mathrm{~min}^{\mathrm{m}}$ \\
\hline \multicolumn{5}{|l|}{ Age $(y)$} \\
\hline $35-44$ & 30 & 46 & 36 & $2 \mathrm{~h} 01 \mathrm{~min}$ \\
\hline $45-54$ & 25 & 36 & 35 & $1 \mathrm{~h} 51 \mathrm{~min}$ \\
\hline $55-64$ & 26 & 35 & 36 & $1 \mathrm{~h} 45 \mathrm{~min}$ \\
\hline $65-74$ & 30 & 39 & 37 & $2 \mathrm{~h} 04 \mathrm{~min}$ \\
\hline \multicolumn{5}{|l|}{ Specific heart complaints } \\
\hline No or few $(0-1)$ & 30 & 37 & 35 & $1 \mathrm{~h} 47 \mathrm{~min}$ \\
\hline Many (3-7) & 25 & 40 & 35 & $1 \mathrm{~h} 45 \mathrm{~min}$ \\
\hline \multicolumn{5}{|l|}{ Pain } \\
\hline Low pain score $(0,1)$ & 30 & 38 & 36 & $2 \mathrm{~h} 13 \mathrm{~min}$ \\
\hline High pain core $(2-4)$ & 25 & 37 & 35 & $2 \mathrm{~h}$ \\
\hline \multicolumn{5}{|l|}{ Killip class } \\
\hline Killip 1 (no LVF) & 30 & 40 & 35 & $2 \mathrm{~h} 04 \mathrm{~min}$ \\
\hline Killip 2-4 (signs of LVF) & $15^{\star}$ & 36 & 35 & $1 \mathrm{~h} 53 \mathrm{~min}$ \\
\hline \multicolumn{5}{|l|}{ Displacement } \\
\hline No & 20 & 33 & 36 & $1 \mathrm{~h} 46$ \\
\hline Yes & $30^{\star}$ & $46^{\star \star \star}$ & 35 & $2 \mathrm{~h} 22^{\star \star \star}$ \\
\hline
\end{tabular}

Mann-Whitney U-Wilcoxon rank sum W test. LVF $=$ left ventricular failure. ${ }^{\mathrm{m}}$ Marginal significant $(\mathrm{p}=0.0572),{ }^{\star} 0.05>\mathrm{p}>0.01,{ }^{\star \star} 0.01>\mathrm{p}>0.001,{ }^{\star \star \star} \mathrm{p}<0.001$. $\mathrm{p}=0.037$ ). When displacement was included in the analysis of variance model the effect of female sex disappeared. Women showed more displacement $(61 \%)$ compared $47 \%$ with men $\left(\chi^{2}=6.0 ; p=0.02\right)$. Patients who attributed their initial complaints to non-cardiovascular causes had a significant longer patient and doctor delay (table 3). Delay times were not related to indicators of size of myocardial infarction as measured by ST increase, CPK maximum values. In the case when the AMI patient was alone when initial complaints began median patient delay was 72 minutes compared with 23 minutes when other people were present (Mann-Whitney $U<0.0001$ ). More social support was related with shorter patient delay.

Within office hours the total delay time was shorter (111 minutes) compared with outside office hours (134 minutes). This was attributable to a significant longer patient delay in out of office hours. Consulting the GP or the locum tenens had no consequences for the delay times. Finally, domicile (urban/rural) was only related to ambulance delay: a difference of seven minutes (urban 32 and rural 39 minutes).

The comparison of the delay times of the patients referred to hospital by a GP compared with the direct call group showed that the direct call group had a substantially lower total delay time: 58 minutes compared with 124 minutes. This difference was explained by the shorter patient delay time of the direct call group (20 minutes compared with 30 ) and, of course, the absence of doctor delay. The GP group and the direct call group did not differ on the variables age, sex, education, and whether or not the infarction occurred in a weekend or in or outside office hours. A trend was found that previous history of AMI was more frequently present in the direct call group ( $21 \%$ compared with $12 \%$ in the GP group; $\chi^{2}$ test, $\mathrm{p}=0.08$ ). The size of AMI was not related to direct call. 
The GP group and the direct call group differed significantly on domicile (urban/rural). In the municipality of Groningen $18 \%$ of the AMI patients directly called an ambulance through the emergency number compared with $5 \%$ of the patients residing in rural counties $\left(\chi^{2}\right.$ test, $p$ $<0.0001)$.

Of all patients three quarters arrived in hospital within six hours after onset of symptoms; $65 \%$ of these patients and another $16 \%$ of the remaining patients received thrombolytic treatment.

\section{Discussion}

This study refers to patients with AMI who were admitted to hospital. No conclusions can be drawn for patients who were not referred to hospital and patients age 75 and older. We recommend for future studies to include patients older than 75 years for three reasons. Firstly, our data demonstrate that living alone is related to longer patient delay. Secondly, life expectancy is increasing and incidence of AMI is high in older age groups. In particular women at old age frequently live alone. Thirdly, age as exclusion criterium for thrombolytic treatment in no longer confined to 75 years.

Despite the overall short delay times, as compared with other studies, ${ }^{16}$ there are some matters of concern. One of them is the sub-group of patients $(21 \%)$ that waited two hours or more before calling a GP. In these cases the doctor delay, and in particular doctor decision time was relatively long. This indicates that these cases were hard to diagnose. As these patients did not differ in number of specific symptoms nor by intensity of pain and showed more displacement of symptoms it is clear that there is no easy solution. Patients who were alone when symptoms started needed much more time to call a GP. Apparently these patients hesitated longer. It should be considered to encourage people to communicate their symptoms with others, in particular when living alone.

Experience from Rotterdam shows that decision making by GPs can be facilitated by pre-hospital diagnosis with a 12 lead electrocardiogram (ECG). Trained ambulance crew can record a 12 lead ECG and a personal computer with decision algorithm can give a clear diagnosis. The GP still needs to decide whether or not referral and pre-hospital thrombolysis is indicated. ${ }^{19}$ The ECG is the most reliable predictor for presence or absence of AMI.

Because a longer treatment delay is related with increased mortality risk women with evolving AMI deserve special attention. Longer pre-hospital delay in women was mainly caused by a shorter doctor decision time in men. Women in our study were slightly older but in this study age less than 75 years was not related to longer treatment delay. The main reason for the longer doctor decision time seems to be the use of the coping mechanism displacement of symptoms, in particular in women. It appears from medical literature that physicians pursue less aggressive diagnostic approaches to identify coronary heart disease in women. ${ }^{20}$ Women are older than men when they present with coronary heart disease. First symptoms in women are more frequent angina and less frequent AMI. ${ }^{21}$ Therefore sensitivity and specificity of cardiac complaints in women are lower. Weaver reported in a large multicentre trial that women have longer pre-hospital delay. ${ }^{11}$ Weaver's suggestion, that experienced symptoms may be less severe in women and their significance less understood by doctors, was confirmed in our study.

As female sex is independently associated with increased mortality ${ }^{22}$ there is sufficient evidence to ask GPs to be more alert in case women present symptoms related to AMI. Recently mobile ECG diagnostic services have been introduced in Groningen that may facilitate diagnosis making, in particular in women.

Outside office hours, patients were inclined to wait longer before calling a GP as compared with the time before calling within office hours. From the interviews it appeared that a psychological barrier exists to wake up a GP at night. This barrier cannot be sustained by the facts: the delay times of both GP as well as the locum tenens were not related to the time of the day. Because a perceived barrier is a "real" barrier anyhow, health education campaigns should pay attention to this topic and GPs could emphasise to patients with CHD that they should not hesitate to call outside office hours.

An issue to be debated in the Netherlands is the topic of direct call or not. As stated before, the GP plays an important part in the Dutch health care system. The GP acts as gate keeper to coordinate referral to medical specialists and to promote efficient use of hospitals. This leads to an additional delay time of 38 minutes, being the median GP delay time in this study. The suggestion that this delay can be avoided by direct calling an ambulance might be misleading in the long run. A direct call is only quicker when it is confined to a small group of patients with high urgency. Just stimulating the general public to use direct call in case of suspicion of an AMI might lead to blockage of the emergency cardiology services, resulting in an overall increase of treatment delay. Therefore it is necessary to stimulate the proper use of a direct call number. Suggested target groups are for example patients who had previous AMI or are at high risk for other reasons or in cases the GP is hard to reach (occupied telephone lines).

An alternative strategy is to promote and develop pre-hospital diagnostic services. ${ }^{19}$ In several studies out of hospital thrombolytic treatment has been proved to be successful in reducing treatment delay. ${ }^{24-27}$ An experiment that was conducted in the city of Groningen was stopped after screening 350 patients and inclusion of only seven patients from prehosptial thrombolysis $(2 \%){ }^{28}$ That study demonstrated that pre-hospital triage may lead to out of hospital time delay for sub-groups of AMI patients and may therefore render no clinical advantages. These investigators concluded that patients with chest pain should be delivered to hospital immediately. However, in several studies hospital delay seemed to be a substantial factor that contributes considerably 
to treatment delay. ${ }^{11}$ Depending on the situation (that is, administering thrombolytic treatment in an emergency room or treated in a coronary care unit) hospital delay appeared to vary between 30 and 80 minutes respectively. ${ }^{23}$ In a large scale study $(\mathrm{n}=42021)$ Weaver et al found a medium hospital delay of 1.0 hour for men and 1.2 hour for women. ${ }^{11}$ In our study the median hospital delay (not available for all patients) was 80 minutes (personal communication, MJL de Jongste). This is in line with findings from the medical literature. It is therefore suggested that reduction of hospital delay may prove to be more efficient and a possible easier attainable strategy for time reduction than in the pre-hospital stage.

Members of the Steering Committee were: Wim van den Heuvel, Professor in Medical Sociology, Northern Centre for Healthcare Research, University of Groningen, Betty Meyboom-de Jong, Professor in Family Medicine, University of Groningen, Willem de Jong, Director of the Municipal Health Service of Groningen, Jan Bleeker, Epidemiologist, Municipal Health Service of Rotterdam, Jan Broer, MD-MPH, Municipal Health Service of Groningen, Harry Crijns, Professor in Cardiology, University Hospital of Groningen, Mike J L DeJongste, Cardiologist, University Hospital of Groningen and Leo Schrijvers, Cardiologist, Martini Hospital Groningen.

The authors wish to thank Mr Joh Schraa (director ambulance dispatch centre Groningen) and Mrs ACouvert (chief ambulance dispatch centre Drenthe) for their participation in this study.

Funding: this study was supported by the Dutch Heart Foundation (Grant NHS-42-011)

Conflicts of interest: none.

1 Boersma E, Maas ACP, Deckers JW, et al. Early thrombolytic treatment in acute myocardial infarction: re-appraisal of the golden hour. Lancet 1996;348:771-5.

2 Hunink MGM, Goldman L, Tosteson ANA, et al. The recent decline in mortality from coronary heart disease, $1980-1990$. The effect of secular trends in risk factors and treatment. JAMA 1997;277:535-42.

3 Yarzebski J, Goldberg RJ, Gore JM, et al. Temporal trends and factors associated with extent of delay to hospital arrival in patients with acute myocardial infarction: The Worcester Heart Attack Study. Am Heart f 1994;128:255Worcest

4 Simoons ML, Serruys PW, Brand M van der, et al. Improved survival after early thrombolysis in acute myocardial infarction. Lancet 1985;ii:578-82.

5 Maynard C, Weaver WD, Lambrew C, et al. factors influencing the time to administration of thrombolytic therapy with recombinant tissue plasminogen activator (Data from the National Registry of Myocardial Infarction). Am f Cardiol 1995;76:548-52.

6 Newby LK, Rutsch WR, Califf RM, et al. Time from symptom onset to treatment and outcomes after thrombolytic therapy. 7 Am Coll Cardiol 1996;27:1646-55.

7 Boersma E, Maas ACP, Deckers JW, et al. Early thrombolytic treatment in acute myocardial infarction: reappraisal of the golden hour. Lancet 1996;348:771-5.
8 Rogers WJ, Bowlby LJ, Chandra NC, et al. Treatment of myocardial infarction in the United States (1990-1993). Observations from the National Registry of Myocardial Infarction. Circulation 1994;90:2103-13.

9 Kereiakes DJ, Gibler WB, Martin LH, et al. Relative importance of emergency medical system transport and the prehospital electrocardiogram on reducing hospital time delay to therapy for acute myocardial infarction: a preliminary report from the Cincinnati Heart Project. Am Heart 7 1992;123:837-9.

10 Boisjolie CR, Sharkey SW, Cannon CP, et al. Impact of a thrombolysis research trial on time to treatment for acute
myocardial infarction in the emergency department. $A m \mathcal{F}$ Cardiol 1995;76:396-400.

11 Weaver WD, White HD, Wilcox RG, et al. Comparisons of characteristics and outcomes among women and men with acute myocardial infarction treated with thrombolytic therapy. $\mathscr{f} A M A$ 1996;275:777-82.

12 Simon $\mathrm{AB}$, Feinleb M, Thompson HK. Components of delay in the prehospital phase of acute myocardial delay in the prehospital phase of acut
infarction. Am $\mathcal{F}$ Cardiol 1972;30:476-82.

13 Tjoe SL, Luria MH. Delays in reaching the cardiac care unit: an analysis. Chest 1972;61:617-21.

14 Rawles JM, Haites NE. Patient and general practitioner delays in acute myocardial infarction. BMF 1988;296:882-4.

15 Hart HN. Hartbewaking in de prehospitale fase (Coronary care in the prehospital phase). [Thesis]. Rotterdam: Erasmus University, 1978 .

16 Bleeker J, Simoons ML, Erdman, RAM, et al. Patient and doctor delay in acute myocardial infarction: a study in Rotterdam, the Netherlands. Br f Gen Pract 1995;45:181-4.

17 Beunderman R. Patientvertraging en het herkennen van hartinfarctverschijnselen (Patient delay and the recognition of symptoms of myocardial infarction). Ned Tijdschr Geneeskd 1976;120:374-8.

18 Bär FW, Vermeer F, de Zwaan C, et al. Value of admission electrocardiogram in predicting outcome of thrombolytic therapy in acute myocardial infarction. Am f Cardiol 1987; therapy in 5 : 13 .

19 Greijseels EWM. Prehospital triage to improve diagnostic and therapeutic decisions in patients with suspected
myocardial infarction. [PhD thesis]. Rotterdam: Erasmus University, 1994.

20 Steingart RN, Packer M, Hamm P, et al. Sex differences in the management of coronary artery disease. $N$ Engl $\mathcal{F}$ Med 1991;325:226-30.

21 Lerner DJ, Kannel WB. Pattern of coronary heart disease morbidity and mortality in the sexes: a 26-year follow-up of the Framingham population. Am Heart f 1986;111:383-90.

22 Kitler ME. Coronary disease: are there gender differences? Eur Heart f 1994;15:409-17.

23 Birkhead JS on behalf of the joint audit committee of the British Cardiac Society and a cardiology committee of the Royal College of Physicians of London. Time delays in provision of thrombolytic treatment in six district hospitals. provision of thrombolytic

24 The European Myocardial Infarction Project Group. Prehospital thrombolytic therapy in patients with suspected acute myocardial infarction. $N$ Engl $f \mathrm{Med}$ $1993 \cdot 329 \cdot 383-9$

25 GREAT Group. Feasibility, safety, and efficacy of domiciliary thrombolysis by general practitioners: Grampian region early anistreplase trial. $B M \mathcal{F} 1992 ; 305: 548-53$

26 Bouten MJM, Simoons ML, Hartman JAM, et al. Prehospital thrombolysis with alteplase (rt-PA) in acute myocardial infarction. Eur Heart $\mathcal{F}$ 1992;13:925-31.

27 Weaver WD, Cerquiera M, Hallstrom AP, et al, for the myocardial infarction triage and intervention project group. Prehospital-initiated vs hospital initiated thrombolytic therapy - The myocardial infarction triage and intervention (MITI) trial. fAMA 1993;270:1211-16.

28 Brügemann J, van der Meer J, de Graeff PA, et al. Logistic problems in prehospital thrombolysis. Eur Heart f 1992;13: $787-8$. 